Journal of Applied Fluid Mechanics, Vol. 15, No. 2, pp. 373-385, 2022.

Available online at www.jafmonline.net, ISSN 1735-3572, EISSN 1735-3645.

https://doi.org/10.47176/jafm.15.02.33004

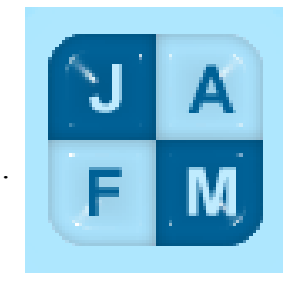

\title{
Particle Image Velocimetry Investigation of the Flow for the Curved Type Wind Turbine Shroud
}

\author{
A. Ilhan ${ }^{1}$, S. Tumse ${ }^{2}$, M. Oguz Tasci ${ }^{2}$, M. Bilgili ${ }^{3}$ and B. Sahin ${ }^{2 \dagger}$ \\ ${ }^{1}$ Ankara Yildirim Beyazit University, Faculty of Engineering and Natural Sciences, Department of \\ Energy Systems Engineering, Kecioren, Ankara, 06010, Turkey \\ ${ }^{2}$ Cukurova University, Engineering Faculty, Mechanical Engineering Department, Adana, Turkey \\ ${ }^{3}$ Cukurova University, Ceyhan Engineering Faculty, Mechanical Engineering Department, Ceyhan, Adana, \\ 01950, Turkey \\ †Corresponding Author Email: bsahin@cu.edu.tr
}

(Received May 12, 2021; accepted September 17, 2021)

\begin{abstract}
Determining the flow structure in the flanged diffuser shrouding designed to be used for a wind turbine, plays a vital role in improving efficiency in small-scale wind turbine technology. In this study, the flow structure in the curved type flanged diffuser was investigated in terms of water flow velocity increase, by using particle image velocimetry $(P I V)$ measurements. The dimensionless magnitude of the resultant velocity determined at downstream and up radial regions as well as at the flange downstream of the curved type wind turbine shroud, revealed that resultant velocity was increased by a factor of 1.5 . This increase of the wind velocity will result the wind energy interacting with the rotor blades to be enhanced by 3.38 times more. By the designed shrouding component to be used in micro wind turbines, it is also aimed to start the power generations in these types at a lower value of cut-in wind speed value.
\end{abstract}

Keywords: Flow velocity enhancement; Particle image velocimetry; Wind power generation.

\section{INTRODUCTION}

Wind power generation systems have been in use more than 3000 years, and several different types of wind-mills have been invented in this time period, which started by the initial appearance of the ancient Persian vertical axis wind-mills. However, wind power generation systems, in a real sense, have been started to be used in electrical power generations by late 1800 s and early $1900 \mathrm{~s}$. But, wind turbines mainly started to spread across the World in the last three decades (Sørensen 2011).

On the other hand, a wind turbine generates power from the wind with the cube of the approaching wind speed. The relation of the power generation according to the approaching free-stream wind speed, is given by the equation, $P_{a v}=0.5 \rho \mathrm{A}_{\text {rotor }} \mathrm{UD}^{3}$. In this equation, the abbreviations of $\rho, A_{\text {rotor }}$, and $U_{D}$, respectively denote the air density, the rotor blade cross-section area, and wind speed on the rotor disc. The wind speed on the rotor disc is the induced freestream wind speed by the axial flow induction factor. This equation presents maximum theoretical wind power that is available to be converted into electric power.
The capacity of wind turbines increased significantly in last two decades, and they were classified with different categories by Tummala et al. (2016), as it is presented in Table 1. The leading companies have started to manufacture wind turbines with the capacities of 7.5-8 MW. It is also known that there are as well projects for developing wind turbines for offshore applications with the huger power capacities of 10-20 MW.

According to the Betz limit, the percentage of the maximum possible ideal conversion of wind power is given by $C_{p \max }=0.593$ (Bilgili et al. 2015). Accordingly, there have been several different designs of conventional wind turbines which cannot perform the power generations over the Betz limit, as it is seen in Fig. 1 (Hau 2006).

A minor rise in approaching wind speed to rotor can induce a large increase in wind power generation. For that reason, developing new technologies that can considerably accelerate the approaching wind speed is the desired fact. In this respect, augmenting wind turbines with a diffuser component (DAWT) was first proposed by Lilley and Rainbird (1956) in 1956 year. This work was followed by many other studies, such as Oman et al. (1975), Igra (1977), 
Table 1 Classification of horizontal axis wind turbines categorized by the rotor diameters and power rating.

\begin{tabular}{|c|c|c|c|c|c|c|c|}
\hline \multicolumn{2}{|c|}{ Type of wind turbine } & \multicolumn{2}{|c|}{ Rotor diameter $(m)$} & \multicolumn{2}{|c|}{ Swept area $\left(m^{2}\right)$} & \multicolumn{2}{|c|}{$\begin{array}{l}\text { Standard power rating } \\
(k W)\end{array}$} \\
\hline & & Min. & Max. & Min. & Max. & Min. & Max. \\
\hline \multirow{3}{*}{$\begin{array}{l}\text { Small } \\
\text { scale }\end{array}$} & Micro & 0.5 & 1.25 & 0.2 & 1.2 & 0.004 & 0.25 \\
\hline & Mini & 1.25 & 3 & 1.2 & 7.1 & 0.25 & 1.4 \\
\hline & House-hold & 3 & 10 & 7.1 & 79 & 1.4 & 16 \\
\hline \multicolumn{2}{|c|}{ Small commercial } & 10 & 20 & 79 & 314 & 25 & 100 \\
\hline \multicolumn{2}{|c|}{ Medium commercial } & 20 & 50 & 314 & 1,963 & 100 & 1,000 \\
\hline \multicolumn{2}{|c|}{ Large commercial } & 50 & 100 & 1,963 & 7,854 & 1,000 & 3,000 \\
\hline \multicolumn{2}{|c|}{ Largest commercial } & 100 & 160 & 7,854 & 20,106 & 3,000 & 8,000 \\
\hline \multicolumn{2}{|c|}{ Under development } & 160 & 220 & 20,106 & 38,013 & 8,000 & 20,000 \\
\hline
\end{tabular}

Foreman et al. (1978), Gilbert et al. (1978), Igra (1981), Gilbert and Foreman (1983), Phillips et al. (2002), Hansen et al. (2000), Bet and Grassmann (2003), Jafari and Kosasih (2014), Shonhiwa and Makaka (2016), Ilhan et al. (2018), Ilhan et al. (2021a), and Ilhan et al. (2021b). On the other hand, particle image velocimetry $(P I V)$ analyzes performed on similar subjects are as follows: Herges et al. (2014), Abraham et al. (2019), Li et al. (2020), Hollands et al. (2020), Dar and Porte-Agel (2020), Tumse et al. (2021), Inman et al. (2021), Doan et al. (2021).

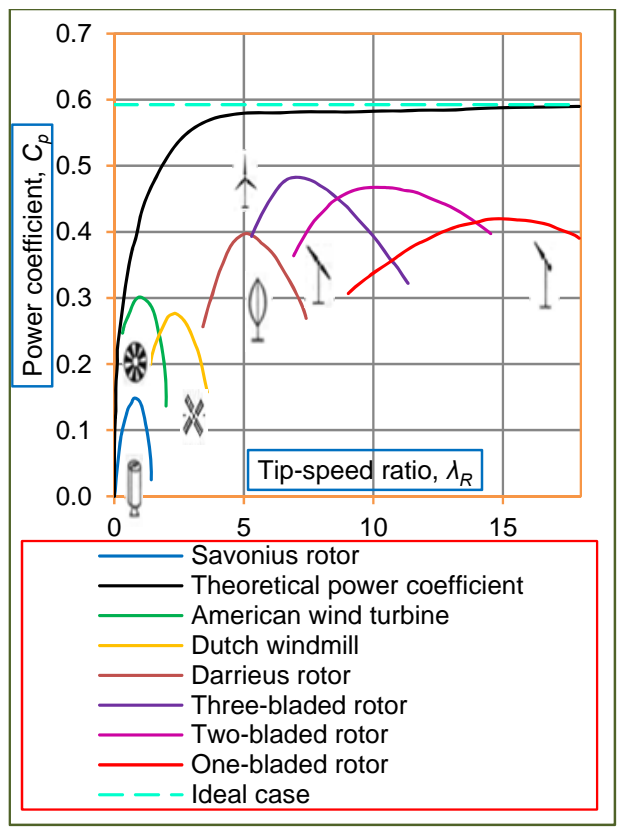

Fig. 1. Power coefficient, $C_{p}$ ranges of different wind turbine designs (Hau 2006).

In this table, the classification of wind turbines has been shown by subcategories. The classifications has been defined in this table by subcategories to include small scale, small commercial, medium commercial, large commercial, largest commercial, and under development. Besides, the identification of these six subcategories has been specified, based on the minimum and maximum values of, rotor diameters, rotor swept areas, as well as the power rating values. To explain more clearly, small commercial wind turbines include rotor diameter values to be greater than $10 \mathrm{~m}$ and less than $20 \mathrm{~m}$. Besides, its corresponding swept area will be greater than $79 \mathrm{~m}^{2}$ and less than $314 \mathrm{~m}^{2}$. Finally, this classified type will have power rating values to be greater than $25 \mathrm{~kW}$ and less than $100 \mathrm{~kW}$. Exceeding the maximum values of the ranges will result the definition of the upper subcategory, i.e., the medium commercial type for this case. On the other hand, as it is moved beyond the minimum values of rotor diameter, swept area, and standard power rating, small scale type will be obtained in this case. Small scale type of wind turbines have further three subcategories named as micro, mini, and household types, and their range identifications have been also demonstrated in this table.

Shrouding of wind turbines is one of the most important ways of increasing the generated output power and reducing the cost of power generation. That is to say, an important way of enhancing the wind turbine output power under low wind speed can be achieved through concentrating of airflow by a proper shrouding geometry. This geometry configuration involves casing of the rotor with diverging and converging ducts to convey the air mass flow rate through the wind turbine at a higher rate. Studies demonstrated that better performance under low free-stream wind speed, $U_{\infty}$ was observed with the utilization of the concentrating shroud compared to a conventional horizontal or vertical axis wind turbine (Shonhiwa and Makaka 2016).

Innovative and original aspects of ongoing research works focus on ensuring the optimization of a casing system around the wind turbine. The casing system consists of components including nozzle-diffuserflange combination or a lens is used in order to enhance the performance of small scaled conventional wind turbines having a moderate capacity. 
In the case of nozzle-diffuser-flange applied casing system, instead of having a straight diffuser wall, it is better to have a curved wall. This method also provides an inlet section as well as it works in the form of an inlet nozzle in the contracting section, besides the curved shrouding performs best in terms of the velocity enhancement. In this regards, Ohya and Karasudani (2010) combined a shorter curved casing with a flange at the outlet periphery of the casing and also formed a curved nozzle as inlet shrouding at the entrance of the casing, as seen in Fig. 2. This flange component also named as "brim" develops vortices and shedding of these vortices causes a low-pressure region downstream of the casing, and curved nozzle at the entrance results in a higher mass flow rate of air running through the wind turbine rotor blades (Wang et al. 2015).

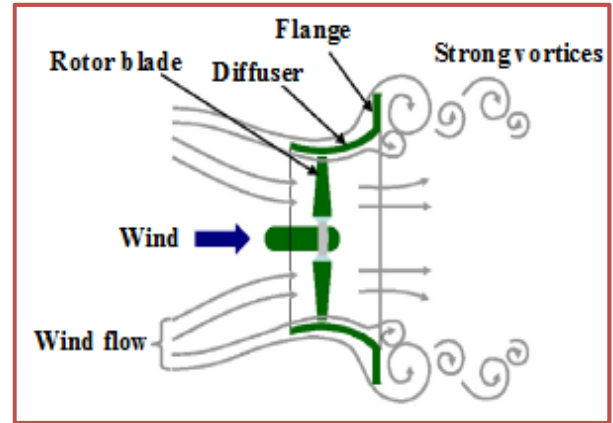

Fig. 2. Illustration of a brimmed curved nozzlediffuser casing structure (Wang et al. 2015).

This study provides the modeling and optimization of a curved type of wind turbine casing body which is studied by particle image velocimetry $(P I V)$. The aim of the study is to demonstrate increase of the flow velocity in the vicinity of the shrouding body, to reveal theoretical boost of wind turbine power generation. So, the main purpose is to obtain a proper shrouding surrounding the turbine rotor disc for micro wind turbine power generation.

Further experimental $P I V$ analysis studies for wind turbine shrouding systems mainly available in the literature include studies of Ohya and Karasudani (2010), Abe et al. (2005), Ohya et al. (2008), Chen et al. (2012), Kosasih et al. (2012), Kosasih and Tondelli (2012), Chen et al. (2013), Wang et al. (2008), Hayashi et al. (2005), Rio Vaz et al. (2014), Chang et al. (2014), Setoguchi et al. (2004), Fletcher et al. (2007), Coşoiu et al. (2013), Abe and Ohya (2004), Mansour and Meskinkhoda (2014), Ohya et al. (2006), Scherillo et al. (2011), Coiro et al. (2009).

The $P I V$ system is a very effective measurement method for separated and non-steady flows, since it can generally measure instantaneous velocity values within a given area at many points at the same time. Measurement of instantaneous velocity values and analysis of measured velocity values and turbulence statistics provide information about the physics of flow. The novelty of the study is to present a quantitative flow analysis of a flow field instantaneously using the PIV technique for the considered wind turbine diffuser shroud, performed in a water channel, instead of using a wind tunnel in order to have more accurate results. Furthermore, it is crucial to display the vorticity-dominated separated flow regions using the PIV method quantitatively. In particular, it is possible to give further information about the physics of the turbulent flow structures by quantitatively displaying the instantaneous velocity flow field. Similarly, there are only numerical studies and qualitative observations in the literature. As it is known, it is possible to produce the most reliable data experimentally. For this reason, providing the experimental data obtained by the quantitative observation of the separated flow regions to the literature can contribute to the design and research studies.

\section{MATERIALS AND METHODS}

Particle Image Velocimetry and dye flow visualization $(P I V)$ experiments were conducted in a free-surface water channel of fluid mechanics laboratory of Cukurova University. The water channel used for this purpose has dimensions of $8,000 \mathrm{~mm} \times 1,000 \mathrm{~mm} \times 750 \mathrm{~mm}$. This water channel is manufactured of $15 \mathrm{~mm}$ thick transparent plexiglass sheet including upstream and downstream fiberglass reservoirs. The water was pumped through a settling chamber and traveled through a honeycomb section of $2: 1$ channel contraction just before arriving the test chamber at downstream. The turbulence intensity was kept below $0.4 \%$ by the utilization of the reservoirs and honeycomb screen arrangements. The pumping of water was executed by an electric motor including a variable speed controller. In the study of Ozturk (2006), it was stated that the maximum boundary layer thickness of this water tunnel was $6 \mathrm{~cm}$. In this laboratory, the images were received from CCD (charged-coupled device) camera which had a pixel resolution of $1,600 \times 1,200$ and equipped with a $60 \mathrm{~mm}$ focallength lens. The flow was fed by hollow spherical particle having same density with water. For this reason, these particles were suspended in the water. Moreover, the diameter of the silver-coated particle was $12 \mu \mathrm{m}$. The instantaneous flow images were obtained at a frequency of $15 \mathrm{~Hz}$. The dynamic studio V4 software was used to obtain images, and the software performed stereo and adaptive correlations to synchronize the system. Crosscorrelation technique of $32 \times 32$-interrogation window was operated with an overlap of $50 \%$, to provide Nyguist criterion in the velocity field determination. For each run, 99x74 grid was obtained, so in total 7,326 velocity vectors were acquired for each instantaneous image. Besides, the illumination of the flow field was obtained by two pulsed Neodymium-doped Yttrium Garnet (Nd:YAG) laser having a wavelength of $532 \mathrm{~nm}$. Each laser source had a $120 \mathrm{~mJ}$ maximum energy output. The thickness of the laser sheet was adjusted as $1.5 \mathrm{~mm}$. Time retardation between frames was $1,750 \mu \mathrm{s}$. The time interval between pulses was adjusted with regard to the structure of vortical flow. In the study of Raffel et al. (2018), it was expressed 
that if the time interval is not adjusted correctly, the number of bad vectors increases. Moreover, due to some material and system problems such as the change in particle size, particle overlaps, noise of electronic devices; the uncertainty takes place in velocity measurements (Hart 2000). Westerweel (1993) who carried out $P I V$ experiments in the same conditions with this study has revealed that the velocity measurements have an uncertainty which is less than $2 \%$. In the case of dye experiments; flow characteristics of the wind turbine casing were visualized using a fluorescent dye. This dye shines under the laser sheet, and it was utilized in creating color alterings of water. The dye was placed in a small sized container which was arranged in a place of $500 \mathrm{~mm}$ above the water channel free surface. Dye was injected at downstream region of wind turbine casing body. In order to capture video images obtained for vortex flow configurations properly, SONY HD-SR1 video camera having $24 \mathrm{~Hz}$ frequency was utilized. The software of this video camera, referred as frame grabber, was used for capturing of these images. Although visualizations obtained by dye utilizations give no numerical information about vorticity flow structure, it presents a brief and rough idea about the structure of the water flow over the wind turbine casing body. During the instantaneous velocity measurements with $P I V$ method, the field of view was adjusted as $87 \mathrm{~mm} \mathrm{x}$ $65 \mathrm{~mm}$, which makes the spacing between vectors as $0.87796 \mathrm{~mm}$, thus 3.3 pixel in both $\mathrm{x}$ and $\mathrm{y}$ measurement planes.

In Fig. 3, the image of the iron profile welded to fix the shroud system and the hub profile to the channel has been shown. The casing profile was fixed with the iron profile using screws at both sides, as it is shown in this figure. In addition, the shroud profile and the hub were fixed by using four laths having $90^{\circ}$ of angles between them.

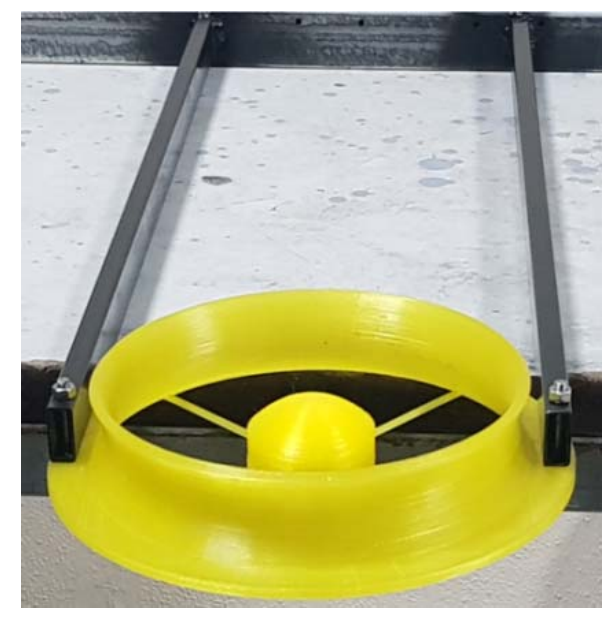

Fig. 3. The studied geometry.

The wind turbine shroud types generally available in the literature are shown in Fig. 4c (types 1, 2, and 3). This figure indicates whole optimized geometrical parameters required in the design procedures. Namely, this figure has been presented both to demonstrate the existing shrouding types in the literature and show all geometric parameters, including these reported three types of geometries. Only the geometric formation of these three configurations is different, but the geometric parameters correspond to the same concept. For instance, $D_{l}$ refers to the inlet diameter of the shroud independent of the type. Similarly, $D_{2}$ stands for the outlet diameter of the shroud. The minimum crosssectional area inside the casing where the wind turbine is installed is abbreviated by symbol $D$. $D_{\text {rotor }}$ indicates the diameter of the wind turbine rotor in the case of wind turbine utilization inside the casing. The height of the flange is given by $H$. The nozzle, the diffuser, and the whole axial lengths of the casings are respectively denoted by $L_{l}, L_{y}$, and $L$. The largest outlet diameter downstream, including the entire flange height, is $D_{3}$. The abbreviations $D_{h}$ and $L_{s}$ correspond to turbine hub diameter and turbine axial length including the whole mechanical mechanism. Finally, the nozzle contraction and diffuser expansion angles are represented by $\gamma$ and $\theta$, respectively. Finally, the symbol $U_{\infty}$ in Fig. $4 \mathrm{c}$ is the free-stream speed (in this experiment, water) approaching the turbine rotor disc. On the other hand, while the angle information is generally required in the linear type of shrouding shown in Fig. $4 \mathrm{c}$ (type 1), it is generally not required in curved and airfoil types of shroudings. In a curved type of wind turbine shrouding (type 2), the required optimization parameters are generally $D_{1}, D_{2}, D, D_{\text {rotor }}, L_{1}, L_{y}, L$, $H$, and curve path (i.e., the equation). The curve path can be indicated by the mathematical equation of the outer curvature in the structure that has been obtained as,

$$
\begin{aligned}
& x^{2}-28.457844250 . x+202.462224839-y^{2}+ \\
& 194.178216238 . y-9426.294915343=R^{2}
\end{aligned}
$$

The precision of the geometrical dimensions was calculated with high precision. The number of significant digits after the decimal point has been taken as three digits. The design of the profile was not done with any ordinary solid modeling design program. The design was completely realized in Excel within the framework of mathematical and analytical considerations. Then, the designed geometric profile was transferred to the threedimensional solid modeling program. Finally, it was constructed with a $3 \mathrm{D}$ printer using a CAM program.

The origin of the $x$ and $r$ axes on Fig. 4a has been shown with a small coordinate system. The study of Wang et al. (2015) was used in the optimization of our profile. Considering this study, a scale factor of 17.12 was used to optimize the whole dimensions of the casing structure, including $D_{1}, D, D_{\text {rotor }}, D_{2}, H$, $L_{1}, L_{y}, L$, and $D_{3}$. The scale factor was used to work with the geometry properly inside the water channel. In order to perform the experiments properly with the geometry placed inside the water channel, the scale factor was used. Since the geometry of the water channel is of certain dimensions, the proper scale factor was absolutely necessary for the designed profile to fit in the channel. The center of the 


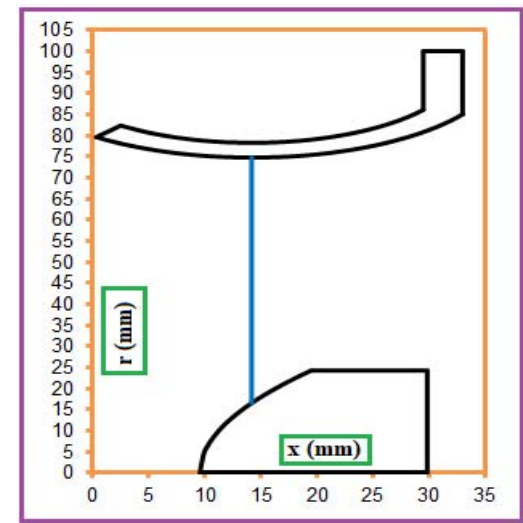

(a)

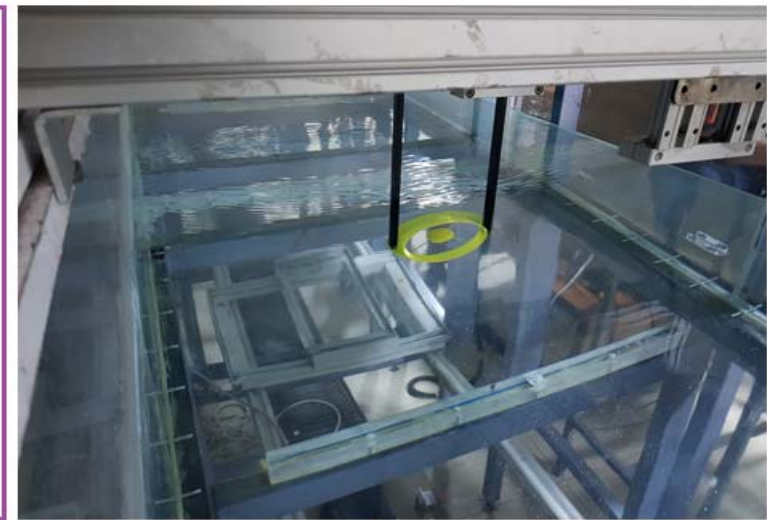

(b)

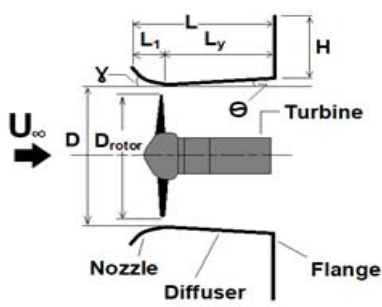

(1)

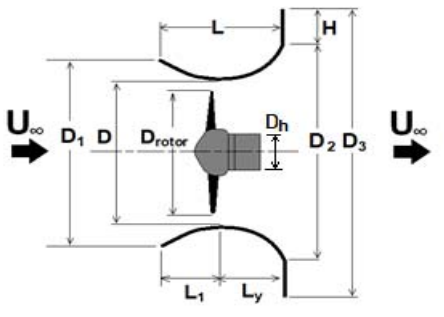

(2)

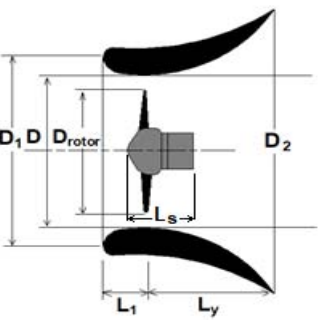

(3)

(c)

Fig. 4. Geometry of the curved type of casing configuration used for PIV analysis (Ilhan et al. 2021a).

curvature was placed to stay in the same vertical line with the wind turbine location diameter, $D$. Principles of the circle analytic geometry was used in the design. Based on circle analytical geometry, two unknowns were initially faced. These unknowns were to correspond to the location of the center of the curvature with respect to the apse and ordinate. However, two equations were derived using two known point information. Considering Fig. 4a, $\mathrm{x}=\mathrm{x}_{\min }$ and $\mathrm{x}=\mathrm{x}_{\max }$ point information was initially determined. The corresponding ordinate values of those points were also determined. Namely, at $\mathrm{x}=\mathrm{x}_{\min }, \mathrm{r}=\mathrm{D}_{1} / 2$; and $\mathrm{x}=\mathrm{x}_{\max }, \mathrm{r}=\mathrm{D}_{2} / 2$ were initially determined. Using a simple code in EES resulted in the apse and ordinate information of the center of curvature of the profile because now we had two equations and two unknowns. Besides, the information of the center location being in the same vertical line with the turbine location diameter, yielded easily determination of the radius size of the curvature.

As soon as the location of the center of curvature and the radius size were found, the circle equation of the profile was determined. This was followed by the operation of the whole body length of the casing, to be divided into one hundred equal parts, and the corresponding $y$-values were finally calculated, within the principles of analytical geometry of the circle. The thickness of the profile was chosen as 3.5 $\mathrm{mm}$, in accordance with the scientific studies. Since the scientific precision of the design was quite high, but the use of high precision in a 3D printer would not be very appropriate; it was decided to use the precision up to three digits after the decimal point and to specify it in this way in the article. The mathematical equation of the outer curvature in the structure that has been obtained as,

$$
\begin{aligned}
& \mathrm{x}^{2}-28.457844250 \mathrm{x}+202.462224839-\mathrm{y}^{2}+ \\
& 194.178216238 . \mathrm{y}-9426.294915343=\mathrm{R}^{2} .
\end{aligned}
$$

Besides, the hub was designed in a similar approach considering the dimensions provided in the study of Kosasih et al. (2012).

In the PIV test section, the casing designed to be used for shrouding of a micro wind turbine, was set in parallel to the water flow. The shrouding component was manufactured of $3.5 \mathrm{~mm}$ thickness. The casing hub center was placed $500 \mathrm{~mm}$ down, with respect to the upper side of the channel. And, it was located 265 $\mathrm{mm}$ down from the free-surface of the flow. Besides, the total height of the flowing water over shrouding body was adjusted to a level of $530 \mathrm{~mm}$; and the wind turbine casing component was positioned just in the middle of the water column.

Additionally, it was placed at a far distance at downstream side of the water channel, in order to prevent problems that may arise during the development of the flow. Free-stream water velocity was adjusted to a value of $0.2 \mathrm{~m} / \mathrm{s}$. This value of the water velocity resulted a Reynolds number 


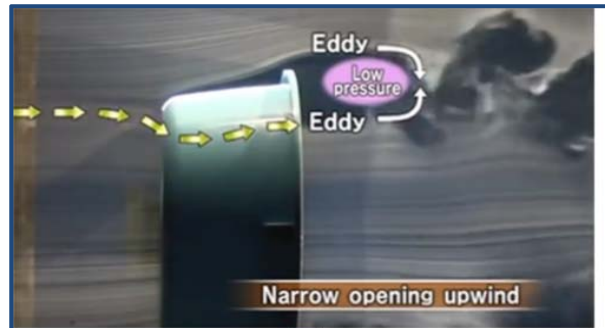

a)

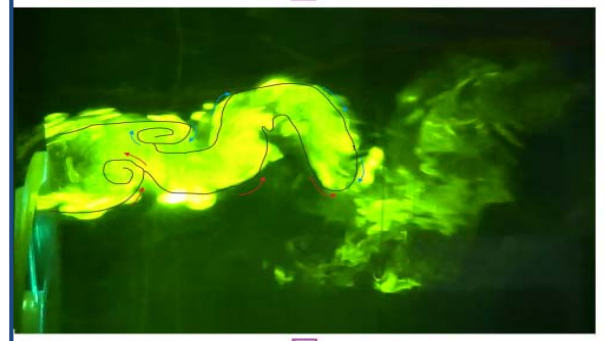

c)

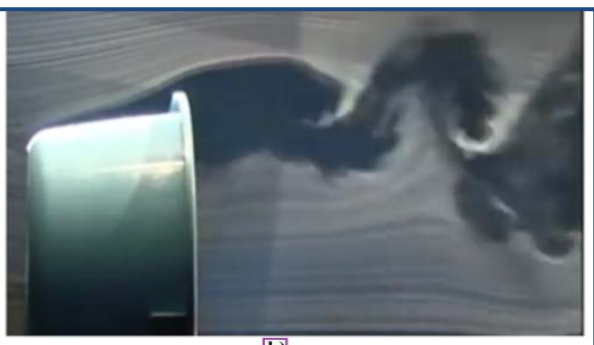

b)

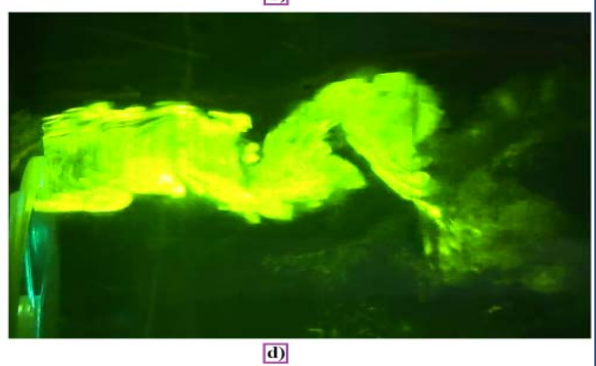

Fig. 5. Low pressure phenomena of up flange downstream images of Ohya et al. (2008), Ohya et al. (2002) and current study, a) up flange downstream dye visualization of Ohya et al. (2008) study, b) up flange downstream dye visualization of Ohya et al. (2002) study, c) first view of up flange downstream dye visualization of the current study, $d$ ) the second view of up flange downstream dye visualization of the current study.

corresponding to $\mathrm{Re}=29,757$ which was determined according to the wind turbine location diameter, $D$. The free-stream water velocity and Reynolds number were both kept constant in PIV and dye experiments. The designed curved type configuration had casing inlet diameter, $D_{1}$, casing exit diameter, $D_{2}$, wind turbine location diameter, $D$, flange height, $H$, turbine hub diameter, $D_{h}$, and turbine axial length including whole mechanical mechanism, $L_{s}$; $159.243,170.023,149.533,14.988,48.501$, and $23.441 \mathrm{~mm}$, respectively. On the other hand, the hub component located inside the casing body was shifted by $9.232 \mathrm{~mm}$ measured with respect to the plane of inlet diameter, $D_{l}$ of wind turbine casing, thus constituting a casing full body length $(L)$ of $L=32.672 \mathrm{~mm}$. In this regards, the optimized configuration setup for the PIV analysis is shown in Fig. 4a (Ilhan 2019).

\section{RESULTS AND DISCUSSIONS}

The dye experiments were conducted in the PIV laboratory of Cukurova University. In these experiments, the dye flow visualization technique was employed for the experiment of flow visualization. The blockage ratio which is the proportion of model's swept area to the crosssectional area of the water tunnel was computed as almost $2 \%$. The blockage corrections were not employed on the results of velocity measurements with PIV method since blockage effects on the experimental outcomes are negligible when it is lower than $10 \%$ as stated by Schreck et al. (2007) and Chen and Liou (2011). The photographic views were obtained considering up flange wake images. Besides, the experiments of Ohya et al. (2008) and
Ohya et al. (2002) were taken into account, to present the similarity of the current results according to the results found in the literature. In these regards, the up flange downstream images of Ohya et al. (2008) and Ohya et al. (2002), which are respectively demonstrated in Fig.s 5a and 5b, present low pressure phenomena of wind turbine shrouding systems. The working principle of this low pressure phenomenon encloses the enhancement of the mass flow rate of the air, interacting with the rotor disc in a wind turbine. Besides, more up flange downstream images obtained from the current study are also shown in Figs. 5c, and 5d; and Figs. 6a, 6b, 6c, and $6 \mathrm{~d}$. The rotary water vorticities were observed at up flange downstream side and their images are demonstrated in these figures. These rotations are the main driving force, which cause the approaching water, near the entrance section, to be quickly inhaled into the diffuser. So, the water which was rapidly inhaled and energized will pass with acceleration along the casing structure.

In the study of Ohya et al. (2008) and Ohya et al. (2002), it was shown that strong vorticity concentrations appear downstream of the flange, causing the extra extraction of the flow of the wind from the wind turbine rotor disc. The large-scale vorticities formed downstream of the flange create a low-pressure region in this flow domain, as shown in Fig. 5a. This similar situation also occurs inside the shroud, in the vicinity of the rotor-blade crosssectional area. According to the first law of thermodynamics, since the total energy is constant and a certain pressure drop occurs by the shroud, the energy of decreasing pressure manifests itself as energy of increasing velocity. Besides, the smoke observation of their studies does not provide 


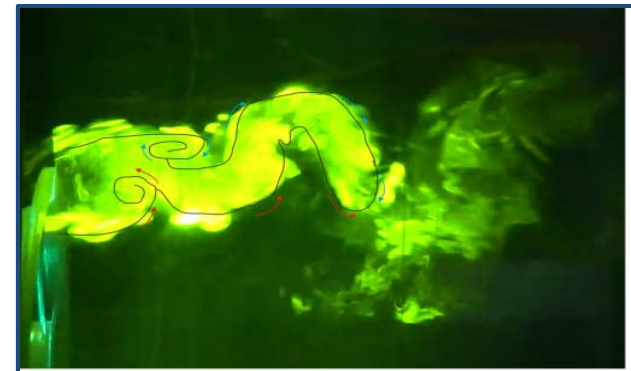

a)

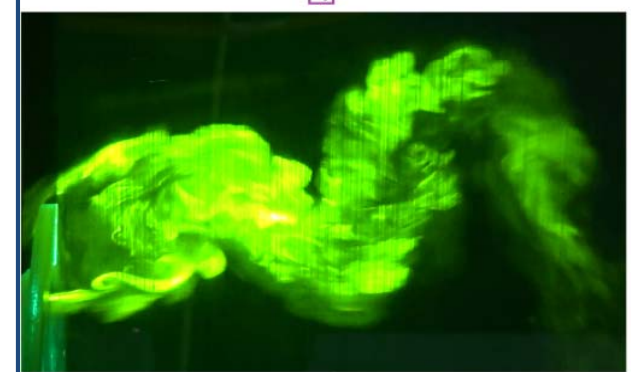

c)

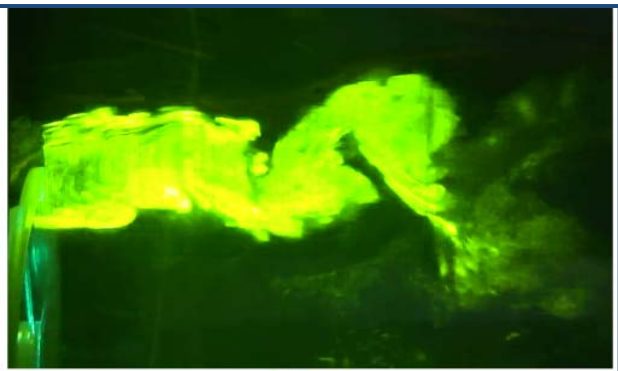

b)

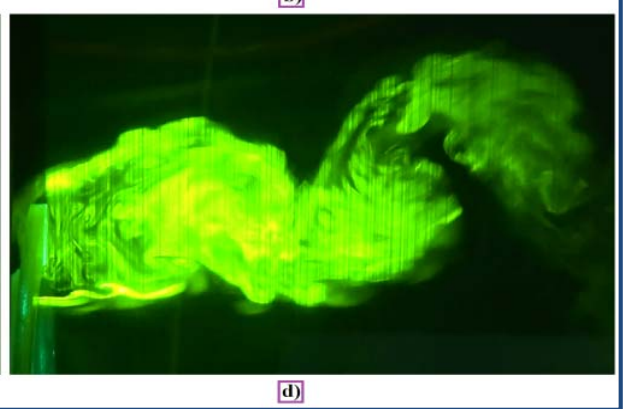

Fig. 6. Low pressure phenomena of up flange downstream images of current study, a) the third view of up flange downstream dye visualization of the current study, b) the fourth view of up flange downstream dye visualization of the current study, c) the fifth view of up flange downstream dye visualization of the current study, d) the sixth view of up flange downstream dye visualization of the current study.

quantitative results about vorticity-dominated flow structures. However, obeying the suggestions of Ohya et al. (2008) and Ohya et al. (2002) provide a significant improvement of the output power of the diffuser augmented wind turbines. Furthermore, a rate of 1.6 enhancements was achieved in the ratio of wind speed when the wind turbine rotor is placed in a specially designed diffuser. Similar large-scale vorticity concentrations in the current study were also obtained quantitatively as shown in Figs. $5 \mathrm{c}, 5 \mathrm{~d}$, $6 \mathrm{a}, 6 \mathrm{~b}, 6 \mathrm{c}$, and $6 \mathrm{~d}$.

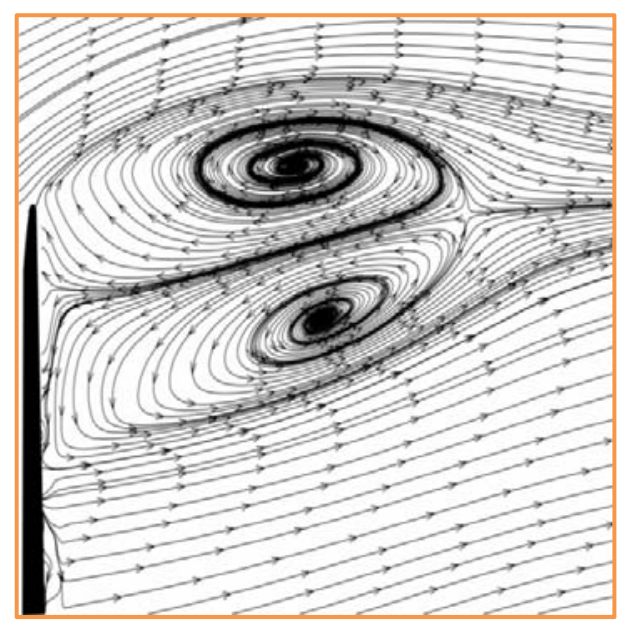

Fig. 7. Flow streamlines at the downstream region and up radial zone of the curved type casing configuration.
Figures 7, 8, and 9 demonstrate axisymmetric flow visualizations at the downstream region of the half section of the shroud.

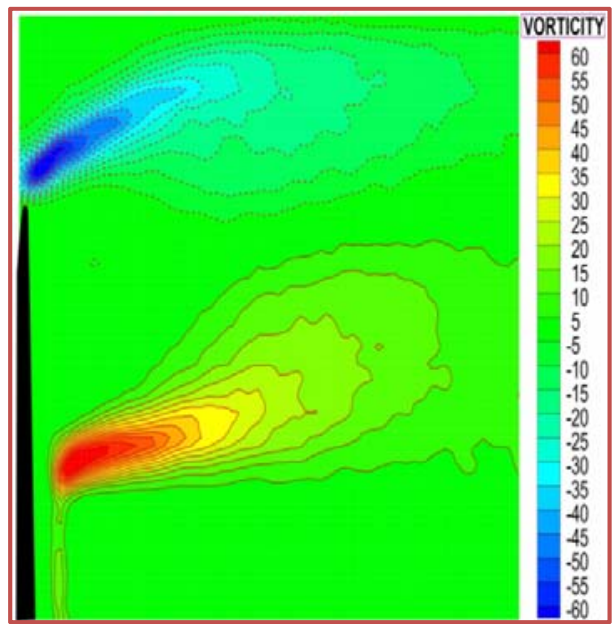

Fig. 8. Vorticity results at the downstream and up radial regions of the curved type casing configuration $(1 / s)$.

Figure 7 indicates flow streamlines at the downstream region and up radial zone of the designed curved type casing configuration. Besides, this figure presents that two large vorticity formations at the downstream regions of this designed curved type of casing configuration were 
formed. These two vorticities are the main driving factors of enhancing mass flow rate of the fluid running through the wind turbine blades. The enhanced mass flow rate through the turbine rotor disc is obtained by the reduced pressure in the vicinity of the turbine rotor disc, causing enhanced flow velocity. To obtain the best performance from the shrouded wind turbine, the proper location of the wind turbine inside the casing is designated by a blue line as shown in Fig. 4a.

Vortex formations at the downstream region and up radial zone of the configuration are demonstrated in Fig. 8. Although strong vortices were obtained at both regions, rotational directions of both were reported to be the inverse. Likewise, Fig. 9 presents wind speed ratio inside and in the vicinity of casing $\left(u / U_{\infty}\right)$; and the wind speed ratio is shown by dimensionless magnitude of the resultant velocity, $|V| / U_{\infty}$ Low wind speed ratios were obtained at downstream of up radial regions intersecting with casing exit diameter $\left(D_{2}\right)$ and intersecting with the exit diameter of the casing including the flange heights $\left(D_{3}\right)$. On the other hand, higher wind speed ratios were obtained at downstream regions intersecting with turbine location diameter $(D)$, due to the functionality of the wind turbine shroud. Additionally, at regions higher elevated than uppermost point of the flange, higher results of the dimensionless magnitude of the resultant velocity, $|V| / U_{\infty}$ were obtained. The increased water velocity in these zones was observed to decrease at further downstream.

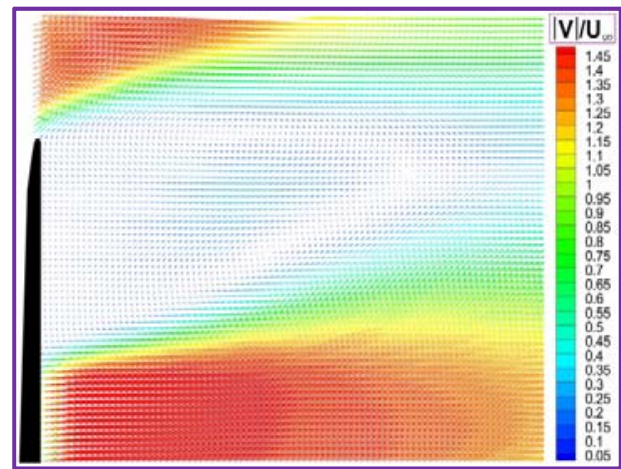

Fig. 9. Dimensionless magnitude of the resultant velocity, $\mid V / U_{\infty}$ at the downstream and up radial regions of the curved type casing configuration.

Axisymmetric flow visualizations of the flange downstream are indicated in Figs. 10, 11, and 12. In these regards, better focused flow visualizations in a specific area are shown. These flow visualizations correspond respectively to the; flange downstream streamline, vorticity, dimensionless magnitude of the resultant velocity, $|V| / U_{\infty}$, which are presented in Figs. 10, 11, and 12 .

Considering Fig. 9, the wind speed increase cannot be referred as locally. On the contrary, the increase in energy that will be useful has been in a wide area. Namely, in the smallest cross-sectional area, that is, in regions where power generations will be obtained, the speed increase was in this direction. In a shrouded wind turbine, the proper location that the wind turbine should be installed is the minimum cross sectional area inside the casing. Considering Fig. 9, as given below, this appears to be the case. Namely, according to Fig. 9, the highest speed increase appears to be in the vicinity of the smallest cross-sectional area, but as well appears over a wide area, as shown in red color.

In this study, no energy generating turbine was used. In addition, due to the testing facilities, it was not possible to view the interior regions of the shroud, which has a minimum cross-sectional area. However, it is a fact that; if the increase in the velocity of the water leaving the shroud in the vicinity of $D_{2}$ diameter is 1.5 , this velocity ratio will be higher at the minimum cross section. This is the case as we do not use turbines. Due to the fact that we did not use a turbine, and since it is known that some of the energy was not converted into mechanical energy on the rotor; depending on the axial flow factor, a little more than the 1.45 of water velocity increase ratio leaving the shroud in the vicinity of $D_{2}$ diameter will occur at the minimum cross section. So, this can be expressed as 1.5. This value of the velocity increase will cause the theoretical increase of power to be 3.38 .

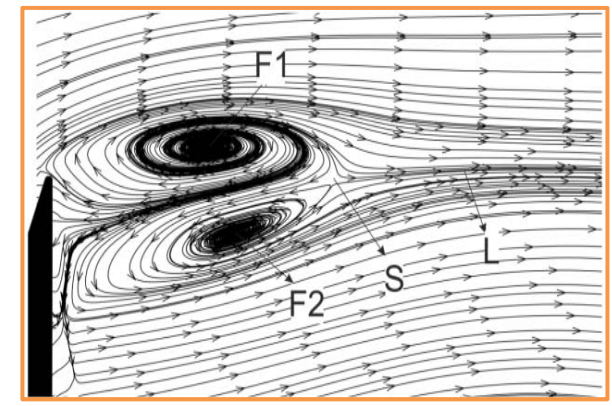

Fig. 10. Streamlines at the flange downstream of the curved type casing configuration.

The designations of $F_{1}, F_{2}, L$, and $S$ are utilized in Fig. 10, for the definitions of the flow characteristics. In this regards, $F$ indicates the foci location; besides, the bifurcation line is abbreviated by $L$ demonstrating the border between wake-flow and free-stream flow regions. Finally, the saddle point in this figure is shortened by $S$. The $S$ takes place at a location above and at downstream region of $F_{2}$. Also, $S$ is positioned at the bifurcation line and located at the just upstream of the furcation end. Due to the curvature and flanged structure of the studied geometry and because of the presence of the hub, two foci are formed. Accordingly, the presence of two foci named as $F_{1}$ and $F_{2}$ result the formation of a bifurcation line, and this line is lengthy in the axial axis $(x)$. Similarly, the presence of two foci and lengthy bifurcation line results a stagnation point, $S$, just in the middle, in which it denotes the location of 
the flow field having zero flow velocity. In this context, the properly designed and optimized curved shrouding structure and hub in the middle generated two big vortices at the immediate downstream of the flange resulting enhanced mass flow rate of water running along the casing. This principle of the physics; will be similarly valid if this configuration is used to surround a wind turbine rotor. In this case, the rotary wind foci will cause enhanced mass flow rate of wind and which finally will result the boost of wind velocity on and in the vicinity of the turbine rotor blades. So, more energized wind will hit the turbine rotor blades, and which will result the turbine rotor disc to rotate at higher rotational speeds. This final situation will cause a significant increase of the stored electric energy on the alternator of the wind turbine.

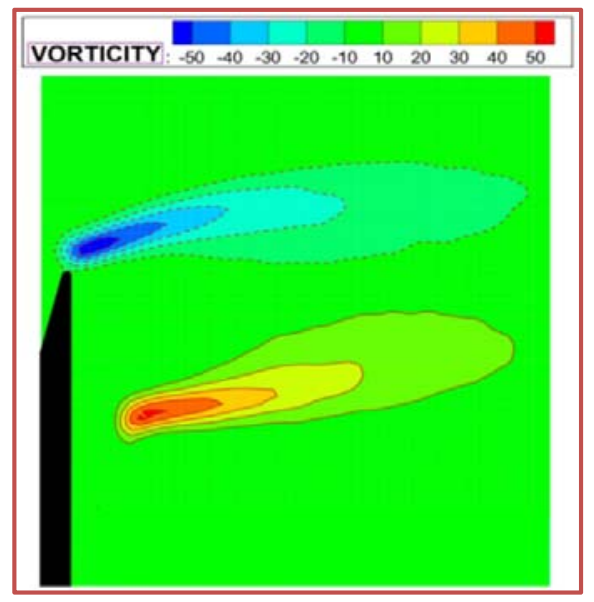

Fig. 11. Vorticity pattern, at the flange downstream, of the curved type casing configuration $(1 / s)$.

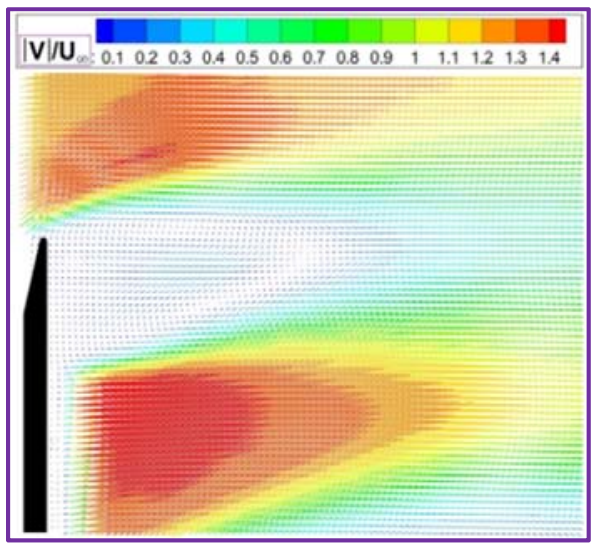

Fig. 12. Dimensionless magnitude of the resultant velocity $\left(|V| U_{\infty}\right)$ at the flange downstream of the curved type casing configuration.

The magnitudes of the swirling regions at the flange downstream of the curved type casing configuration are shown in Fig. 11. Two opposite swirling zones shown in this figure demonstrate that strong vortices are evident. The strong opposite rotary vortices and their magnitudes depend on the curved structure of the casing as well as the flange component designs. Due to the physics of the oppositely rotating water vortices, the dimensionless magnitude of the resultant velocity, $|V| / U_{\infty}$ of water reaches a factor of 1.5 as displayed in Fig. 12. As soon as the pressure inside the casing is reduced, highly energized water molecules passing along the inside of the casing are obtained. These accelerated water molecules in motion are also observed at the immediate downstream of the hub exit zones. At further downstream, the slowing down of the speed of the water molecules occurs; since the speed of the water molecules at further downstream is normalized by the free-stream water flow.

Since the PIV measurement technique can measure the instantaneous velocity vector at more than one point at the same time in a particular flow area, the data obtained facilitates the optimization of the wind turbine casing system parameters. It is possible to determine the characteristic flow changes with time to provide further information about the physics of the flow. As mentioned before, in the study of Ohya et al. (2008) and Ohya et al. (2002), it was shown that well-defined vorticity concentrations appear downstream of the flange, causing the extra extraction of the flow of the wind from the wind turbine rotor disc cross-section. The wind flowing outside the diffuser, called the external flow, separates at the outer edge of the flange and causes the vortex shedding periodically. Since the instantaneous velocity values are known numerically, it is possible to calculate the intensity of these instantaneous swirls or vorticity concentrations and related flow data. As can be seen in Fig. 13, instantaneous patterns of streamlines, distributions velocity vectors, and vorticity concentrations are given. As described earlier, 15 instantaneous flow images are measured per second. Image $f_{l}$ measured at the time of $t$ second, image $f_{2}$ measured at the time of $t+2$ second, and image $f_{3}$ measured at the time of $t+4$ second after starting to capture images given in this figure reveal behaviors of swirling flow. The main goal of this figure is to reveal how the large and small-scale vorticities formed and behaved during the wind bellowing further downstream. The largescale vorticities formed downstream of the flange create a low-pressure region in this flow domain, as shown in Fig. 5a. But, the smoke or dye observations do not provide quantitative results about vorticitydominated flow structures. However, obeying the suggestions of Ohya et al. (2008) and Ohya et al. (2002) provide a significant improvement of the output power of the diffuser augmented wind turbines. Furthermore, the velocity of the wind passing through the diffuser increases 1.6 times due to the geometric structure of the diffuser augmented wind turbine. In addition, the pressure drops in the dead flow region, which is located just downstream of the flange. In this respect, the wind passing through the diffuser passage is directed towards this separated flow region. In addition, the vortex shedding formed at the outer end of the flange also 

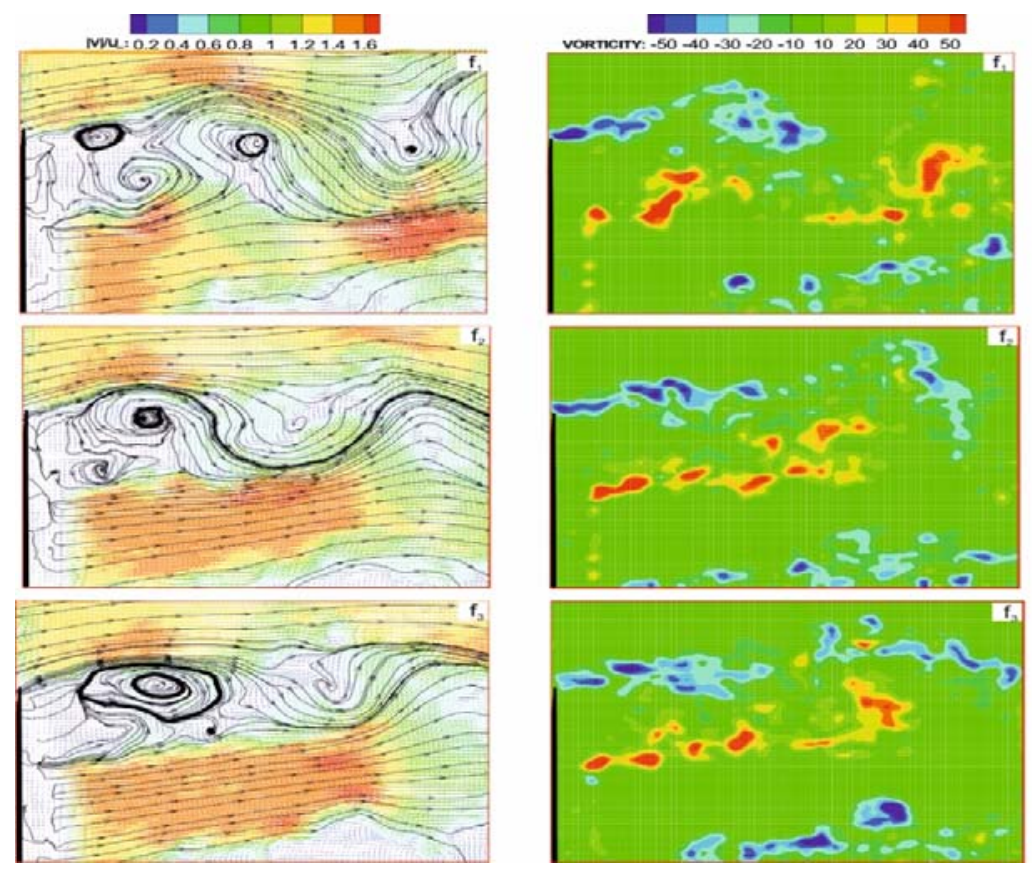

Fig. 13. Instantaneous velocity vectors, streamlines, and vorticity concentrations around the flange.
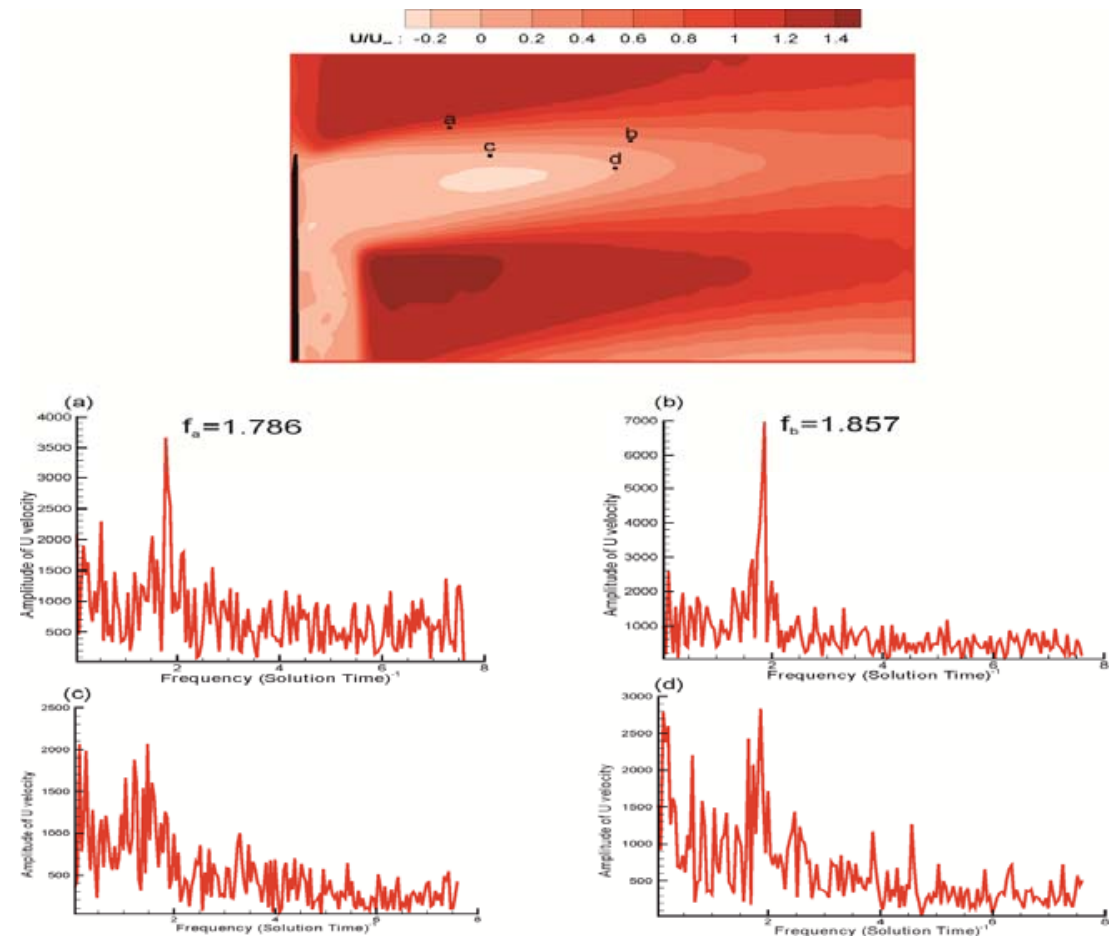

Fig. 14. Spectral analysis of $u$ velocity component at different locations of flow region, $a, b, c$ and $d$ around the flange.

creates an effective swirl in the flow. When the animation of the instantaneous flow data given in Fig. 13 is observed, it is clearly seen that these swirls or well-defined vorticity concentrations also rotate counterclockwise/clockwise, energizing and hence conveying the fluid coming from the diffuser passage in the flow direction further downstream. As a result, the experimental results obtained will also be used to validate the numerical results. It also contributes to the optimization of dimensions of the flanged diffuser casing system for wind turbines.

Figure 14 demonstrates the spectral analysis of $u$ velocity component performed at different locations 
of flow region, $a, b, c$ and $d$ around the flange. The Strouhal number, $S t$ which is associated with an oscillating flow, is denoted as $S t=f D_{1} / U_{\infty}$ where $f$ is the vortex shedding frequency, $D_{l}$ is the diameter of the flange at the inlet section with $D_{l}=0.159 \mathrm{~m}$, and $U_{\infty}$ is the free-stream velocity with $U_{\infty}=0.2 \mathrm{~m} / \mathrm{s}$. As seen in the Figure, whereas the dominant peak frequencies are detected at locations of $a$ and $b$ in the flow region, local peaks are observed at locations of $c$ and $d$ in the flow region. The dominant peak frequencies, $f$ is equal to the $f_{a}=1.786$ and $f_{b}=1.857$ which correspond to the Strouhal number of $S t_{a}=1.422$ and $S t_{b}=1.478$ at locations $a$ and $b$, respectively in the flow field around the flange. The fact that the dominant vortex shedding frequency indicates that the vortex shedding is periodic. On the other hand, the local peak frequencies observed at locations $c$ and $d$ take place as a result of clockwise outer vortices rotating in a clockwise direction and inner vortices rotating in the opposite direction colliding with each other creating small-scale vorticity concentrations.

\section{CONCLUSIONS}

This study reports an optimum designed curved type of wind turbine shrouding having a flange component at downstream and a hub component in the center. The two opposite rotating large foci formed at the immediate downstream regions of the flange components, were obtained due to these designs. The PIV and dye experiments conducted in the fluid mechanics laboratory of Cukurova University, indicated that the water velocity just leaving the shrouding body can be enhanced by 1.5 times.

This experiment demonstrates that in the case of the optimum shrouding body utilization in shrouding of micro wind turbine, the same physics will be valid in terms of the air velocity enhancement. So, the wind velocity enhancement obtained by a factor of 1.5 in a field study can result the wind touching the rotor blades to be theoretically energized by 3.38 times more. Besides, at lower values of free-stream wind speed in the field trial, namely the turbine can start power generation at lower cut-in wind speed values and generated power at the alternator will be significantly increased.

\section{REFERENCES}

Abe, K. and Y. Ohya (2004). An investigation of flow fields around flanged diffusers using CFD. Journal of Wind Engineering and Industrial Aerodynamics 92, 315-330.

Abe, K., M. Nishida, A., Sakurai, Y. Ohya, H. Kihara, E. Wada and K. Sato (2005). Experimental and numerical investigations of flow fields behind a small wind turbine with a flanged diffuser. Journal of Wind Engineering and Industrial Aerodynamics 93(12), 951-970.

Abraham, A., T. Dasari and J. Hong (2019). Investigation of the near-wake behaviour of a utility-scale wind turbine. NAWEA/WindTech 2019 Conference, Amherst, Massachusetts, October.

Bet, F. and H. Grassmann (2003). Upgrading conventional wind turbines. Renewable Energy 28, 71-78.

Bilgili, M., A. Yasar, A. Ilhan and B. Sahin (2015). Aerodynamic characteristics of a horizontal axis wind turbine in Belen-Hatay, Turkey. International Journal of Natural and Engineering Sciences 9(1), 54-58.

Chang, S. H., Lim, Q. H. and K. H. Lin (2014). Design of a wind energy capturing device for a vehicle. $5^{\text {th }}$ International Conference on Intelligent Systems, Modelling and Simulation, Langkawi, Malaysia. January.

Chen, T. Y. and L. R. Liou. (2011). Blockage corrections in wind tunnel tests of small horizontal-axis wind turbines. Experimental Thermal and Fluid Science 35, 565-569.

Chen, T. Y., C. W. Hung and Y. T. Liao (2013). Experimental study on aerodynamics of microwind turbines with large-tip non-twisted blades. Journal of Mechanics 29(3), 15-20.

Chen, T. Y., Y. T. Liao and C. C. Cheng (2012). Development of small wind turbines for moving vehicles: Effects of flanged diffusers on rotor performance. Experimental Thermal and Fluid Science (42), 136-142.

Coiro, D. P., A. De Marco, F. Scherillo, U. Maisto, R. Familio and G. Troise (2009). Harnessing marine current energy with tethered submerged systems: Experimental tests and numerical model analysis of an innovative concept. IEEE International Conference on Clean Electrical Power (ICCEP), Capri, Italy, June.

Coşoiu, C. I., A. M. Georgescu, M. Degeratu and D. Hlevca (2013). Numerical predictions of the flow around a profiled casing equipped with passive flow control devices. Journal of Wind Engineering and Industrial Aerodynamics $114,48-61$.

Dar, A. S. and F. Porte-Agel (2020). Threedimensional wind-turbine wake characterization via tomographic particleimage velocimetry. Journal of Physics: Conference Series 1618(062045).

Doan, M. N., T. Kawata and O. Shinnosuke (2021). Flow Field Measurement of Laboratory-Scaled Cross-Flow Hydrokinetic Turbines: Part IIThe Near-Wake of Twin Turbines in CounterRotating Configurations. Journal of Marine Science and Engineering 9(7), 777.

Fletcher, J., F. Wang, L. Bai, J. Whiteford and D. Cullen (2007). Wind tunnel tests on a wind turbine with contractor and diffuser arrangement. International Conference on Power Engineering, Energy and Electrical Drives, Setubal, Portugal, April, pp. 1-5. 
Foreman, K. M., B. Gilbert and R. A. Oman (1978). Diffuser augmentation of wind turbines. Solar Energy 20, 305-311.

Gilbert, B. L. and K. M. Foreman (1983). Experiments with a diffuser-augmented model wind turbine. Trans. ASME, Journal of Energy Resources Technology 105(1), 46-53.

Gilbert, B. L., R. A. Oman and K. M. Foreman (1978). Fluid dynamics of diffuser-augmented wind turbines. Journal of Energy 2, 368-374.

Hansen, M. O. L., N. N. Sørensen and R. G. J. Flay (2000). Effect of placing a diffuser around a wind turbine. Wind Energy 3(4), 207-213.

Hart, D. P. (2000). PIV error correction. Experiments in Fluids 29, 13-22.

Hau, E. (2006). Wind Turbines: Fundamentals, Technologies, Application, Economics, Springer, Germany.

Hayashi, T., Y. Li and Y. Hara (2005). Wind tunnel tests on a different phase three-stage Savonius rotor. JSME International Journal, Series B 48(1).

Herges, T., D. Bossert, R. Schmitt, D. Maniaci, C. Glen and B. Naughton (2014). Preliminary field test of the wind turbine wake imaging system. 33 ${ }^{\text {rd }}$ ASME Wind Energy Symposium, Kissimmee, Florida, December.

Hollands, E. O., C. He and L. Gan (2020). A particle image velocimetry study of dual-rotor counterrotating wind turbine near wake. Journal of Visualization 23, 425-435.

Igra, O. (1977). Compact shrouds for wind turbines. Energy Conversion and Management 16, 149157.

Igra, O. (1981). Research and development for shrouded wind turbines. Energy Conversion and Management 21, 13-48.

Ilhan, A. (2019). Aerodynamics and Statistical Analyses of Conventional and Diffuser Augmented Wind Turbines, PhD Thesis, Cukurova University, Adana, Turkey.

Ilhan, A., B. Sahin and M. Bilgili (2021b).A review: diffuser augmented wind turbine technologies. International Journal of Green Energy, Accepted,

Ilhan, A., H. Zontul, S. Tumse, M. Bilgili and B. Sahin (2021a). Flow Analyses of Diffuser Augmented Wind Turbines. Energy Sources, Part A: Recovery, Utilization, and Environmental Effects.

Ilhan, A., M. Bilgili and B. Sahin (2018). Analysis of Aerodynamic Characteristics of $2 \mathrm{MW}$ Horizontal Axis Large Wind Turbine. An International Journal of Wind and Structures 27(3), 187-197.

Inman, D., D. G. Cuadrado, V. Andreoli, J. Fisher, G. Paniagua, P. A. N. Aye-Addo, L. Bhatnagar, F. Lozano, T. Meyer and M.
Bloxham (2021). Particle Image Velocimetry in a High-Pressure Turbine Stage at Aerodynamically Engine Representative Conditions. Journal of Engineering for Gas Turbines and Power 143, No. 061031.

Jafari, S. A. H. and B. Kosasih (2014). Flow analysis of shrouded small wind turbine with a simple frustum diffuser with computational fluid dynamics simulations. Journal of Wind Engineering and Industrial Aerodynamics $125,102-110$

Kosasih, B. and A. Tondelli (2012). Experimental study of shrouded micro-wind turbine. Procedia Engineering 49, 92-98.

Kosasih, P. B., N. Bryce, A. Tondelli, and A. Beazley (2012). Experimental study of the performance of bare and nozzle-diffuser shrouded micro wind turbine under axial and non-axial inflow condition. $18^{\text {th }}$ Australasian Fluid Mechanics Conference, Launceston, Australia, December.

Li, C., A. Abraham, B. Li and J. Hong (2020). Incoming flow measurements of a utility-scale wind turbine using super-large-scale particle image velocimetry. Journal of Wind Engineering and Industrial Aerodynamics 197(104074).

Lilley, G. M. and W.J. Rainbird (1956). A preliminary report on the design and performance of ducted windmills. Report 102; The College of Aeronautics Cranfield: Cranfield, UK, April.

Mansour, K. and P. Meskinkhoda (2014). Computational analysis of flow fields around flanged diffusers. Journal of Wind Engineering and Industrial Aerodynamics $124,109-120$

Ohya, Y. and T. Karasudani (2010). A shrouded wind turbine generating high output power with wind-lens technology. Energies 3, 634649.

Ohya, Y., T. Karasudani and A. Sakurai (2002). Development of high-performance wind turbine with brimmed diffuser. Journal of the Japan Society for Aeronautical and Space Sciences 50, 477-482.

Ohya, Y., T. Karasudani, A. Sakurai and M. Inoue (2006). Development of a high-performance wind turbine equipped with a brimmed diffuser shroud. Transactions of the Japan Society for Aeronautical and Space Sciences 49(163), 1824.

Ohya, Y., T. Karasudani, A. Sakurai, K. Abe and M. Inoue (2008). Development of a shrouded wind turbine with a flanged diffuser. Journal of Wind Engineering and Industrial Aerodynamics 96, 524-539.

Oman, R. A., K. M. Foreman and B. L. Gilbert (1975). A progress report on the diffuser augmented wind turbine. Proceedings of the 
A. Ilhan et al. / JAFM, Vol. 15, No. 2, pp. 373-385, 2022.

$3^{\text {rd }}$ Biennial Conference and Workshop on Wind Energy Conversion Systems, Washington, DC, USA, Vol. 8, No. 12, pp. 829-826.

Ozturk, N. A. (2006). Investigation of Flow Characteristics in Heat Exchangers of Various Geometries, PhD Thesis, Cukurova University, Adana, Turkey.

Phillips, D. G., P. J. Richards and R. G. J. Flay (2002). CFD modelling and the development of the diffuser augmented wind turbine. Wind and Structures 5, 267-276.

Raffel, M., C. E. Willert, F. Scarano, C. J. Kahler, S.T. Wereley and J. Kompenhans (2018). Particle Image Velocimetry: A Practical Guide, $3^{\text {rd }}$ ed, Springer, Berlin, Germany.

Rio Vaz, D. A. T. D., A. L. A. Mesquita, J. R. P. Vaz, C. J. C. Blanco and J. T. Pinho (2014). An extension of the blade element momentum method applied to diffuser augmented wind turbines. Energy Conversion and Management 87, 1116-1123.

Scherillo, F., U. Maisto, G. Troise, D. P. Coiro and S. Miranda (2011). Numerical and experimental analysis of a shrouded hydroturbine. IEEE International Conference on Clean Electrical Power (ICCEP), Ischia, Italy, June, pp. 216-222.

Schreck, S. J., N. N. Sorensen and M. C. Robinson. (2007). Aerodynamic structures and processes in rotationally augmented flow fields. Wind Energy 10, 159-178.

Setoguchi, T., N. Shiomi and K. Kaneko (2004). Development of two-way diffuser for fluid energy conversion system. Renewable Energy
29, 1757-1771.

Shonhiwa, C. and G. Makaka, (2016). Concentrator augmented wind turbines: A review. Renewable and Sustainable Energy Reviews $59,1415-1418$.

Sørensen, J. N. (2011). Aerodynamic aspects of wind energy conversion. Annual Review of Fluid Mechanics 43, 427-448.

Tummala, A., R. K. Velamati, D. K. Sinha, V. Indraja and V. H. Krishna (2016). Review on small scale wind turbines. Renewable and Sustainable Energy Reviews 56, 1351-1371.

Tumse, S., M. O. Tasci, I. Karasu and B. Sahin (2021). Effect of ground on flow characteristics and aerodynamic performance of a non-slender delta wing. Aerospace Science and Technology 110(106475).

Wang, F., L. Bai, J. Fletcher, J. Whiteford and D. Cullen (2008).The methodology for aerodynamic study on a small domestic wind turbine with scoop. Journal of Wind Engineering and Industrial Aerodynamics 96, $1-24$.

Wang, W. X., T. Matsubara, J. Hu, S. Odahara, T. Nagai, T. Karasutani and Y. Ohya (2015). Experimental investigation into the influence of the flanged diffuser on the dynamic behavior of CFRP blade of a shrouded wind turbine. Renewable Energy 78, 386-397.

Westerweel, J. (1993). Digital Particle Image Velocimetry, Theory and Application, $\mathrm{PhD}$ Thesis, Delft University Press, Delft, Netherlands. 